\title{
Neurosarcoidosis Mimicking the Recurrence of Malignant Lymphoma
}

\author{
Keishu Murakami ${ }^{a}$ Jinsoo Koha, Junko Taruya ${ }^{a}$ Hidefumi Ito $^{a}$ \\ ${ }^{a}$ Department of Neurology, Wakayama Medical University, Wakayama, Japan; ${ }^{b}$ Department \\ of Neurology, Social Insurance Kinan Hospital, Tanabe, Japan
}

\section{Keywords}

Neurosarcoidosis · Neurolymphomatosis · Sarcoidosis-lymphoma syndrome ·

Mononeuropathy multiplex

\begin{abstract}
A 67-year-old woman with a recurrent history of malignant lymphoma (ML) presented with muscle weakness and paresthesia of the fingertips and feet. Due to the elevated level of serum soluble interleukin-2 receptor and increased ${ }^{18} \mathrm{~F}$-fluorodeoxyglucose uptake in a mediastinal lymph node, neurolymphomatosis was initially suspected. Neurological and electrophysiological examinations were consistent with mononeuropathy multiplex. A diagnosis of neurosarcoidosis was made based on the presence of noncaseating epithelioid granulomas in the mediastinal lymph node, along with the presence of the uveitis, cardiac inflammation, and mononeuropathy multiplex. She was treated with glucocorticoids and azathioprine, and her symptoms disappeared. Sarcoidosis following ML is rare, and since biopsy of nervous systems is often improbable, differentiating neurosarcoidosis and neurolymphomatosis can be difficult as their clinical symptoms can be similar. Clinicians should consider systemic pathological investigations based on ${ }^{18} \mathrm{~F}$-fluorodeoxyglucose positron emission tomography examination in addition to comprehensive evaluation to accurately diagnose neurosarcoidosis.
\end{abstract}

\section{Introduction}

Sarcoidosis is characterized by the formation of noncaseating granulomas in systemic organs [1]. Neurological involvement is present in $5 \%-15 \%$ of patients and can manifest as headache, muscle weakness, sensory disturbance, diplopia, and facial paresis [2]. Due to the 
difficulty in performing a biopsy of the nervous system, the diagnosis of neurosarcoidosis is made based on clinical, laboratory, and lymph node biopsy findings [2]. When neurosarcoidosis manifests as mononeuropathy multiplex with lymphadenopathy, it can be difficult to distinguish from neurolymphomatosis. Serum angiotensin-converting enzyme (ACE) has low sensitivity, and serum soluble interleukin-2 receptor (sIL-2R) level and ${ }^{18} \mathrm{~F}$-fluorodeoxyglucose positron emission tomography $\left({ }^{18} \mathrm{~F}-\mathrm{FDG}\right.$-PET $)$ cannot distinguish between sarcoidosis and malignant lymphoma (ML) $[3,4]$.

In addition, a link between sarcoidosis and ML has been suggested in recent decades. ML occurs 11.5 times more frequently in sarcoidosis patients than in the general population [5]. The development of lymphoma in sarcoidosis patients is known as sarcoidosis-lymphoma syndrome [6], whereas sarcoidosis following ML has been rarely reported [7]. Most cases of sarcoidosis occur within 2 years after ML, but rare cases occur $>10$ years after ML [8].

We report a case of neurosarcoidosis in which neurolymphomatosis was initially suspected because of a history of ML, increased sIL-2R level, and ${ }^{18}$ F-FDG-PET findings. Because neurosarcoidosis may mimic neurolymphomatosis, a comprehensive diagnosis is required.

\section{Case Presentation}

A 67-year-old woman was admitted to the hospital with progressive weakness and paresthesia of the left upper and bilateral lower limbs. She had a history of Hashimoto's thyroiditis and primary biliary cholangitis. Fourteen years prior, she was treated with rituximab-cyclophosphamide-doxorubicin-vincristine-prednisolone for diffuse large B-cell lymphoma of the right breast. She experienced a relapse of ML 12 years prior and received rituximab, which resulted in remission. After long-term remission, an ${ }^{18} \mathrm{~F}$-FDG-PET scan performed 2 years before admission revealed increased uptake of ${ }^{18} \mathrm{~F}$-FDG in the mediastinal lymph node, with a maximum standardized uptake value (SUVmax) of 3.73. One year before admission, she developed weakness and paresthesia in her left fourth and fifth digits, which progressively involved her left second and third fingertips, and then both lower limbs over several months. Three months before admission, she noticed right ophthalmalgia and was diagnosed with uveitis. The ${ }^{18}$ F-FDG-PET scan immediately before admission showed increased SUVmax (5.27) in the mediastinal lymph node. Abnormal ${ }^{18} \mathrm{~F}-\mathrm{FDG}$ accumulation was also shown in the left forearm, right gluteal region, and bilateral lower legs, which were suspected to represent the left ulnar nerve, right superior gluteal nerve, bilateral tibial nerves, and gastrocnemius muscles, respectively (Fig. 1a-c). Laboratory investigation showed progressive elevation of serum sIL-2R. She was admitted to our hospital with suspected neurolymphomatosis.

Neurological examination showed weakness and paresthesia in the left median and ulnar innervation, mild diffuse bilateral weakness of the lower limbs, and distal dominant paresthesia and sensory disturbance of the lower limbs, which were most severe in the area of the right superficial peroneal nerve innervation. Deep tendon reflexes were absent in both lower extremities. These findings were consistent with mononeuropathy multiplex. The remainder of her physical examination was unremarkable. Laboratory testing showed that the serum sIL-2R, ACE, and lysozyme levels were 1,897 (reference range, 127-582) U/ $\mathrm{mL}, 24.8$ (reference range, 7-25) $\mathrm{U} / \mathrm{L}$, and 6.3 (reference range, 5.0-10.2) $\mu \mathrm{g} / \mathrm{mL}$, respectively. The serum calcium level was 9.2 (reference range, 8.8-10.1) mg/dL. Free thyroxine and thyroid-stimulating hormone levels were within the reference range. The T-SPOT.TB test was negative. Cerebrospinal fluid (CSF) analysis showed a slight increase in protein (53 $\mathrm{mg} / \mathrm{dL})$, normal cell count $(3 / \mu \mathrm{L}, 100 \%$ mononuclear cells) with no atypical cells, undetectable sIL-2R $(<50.0 \mathrm{U} / \mathrm{mL})$, and a slight increase in ACE level $(0.7 \mathrm{U} / \mathrm{L} ;$ normal, $<0.5 \mathrm{U} / \mathrm{L})$. The nerve conduction study suggested multifocal demyelination and axonal loss (Table 1):

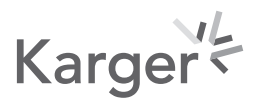




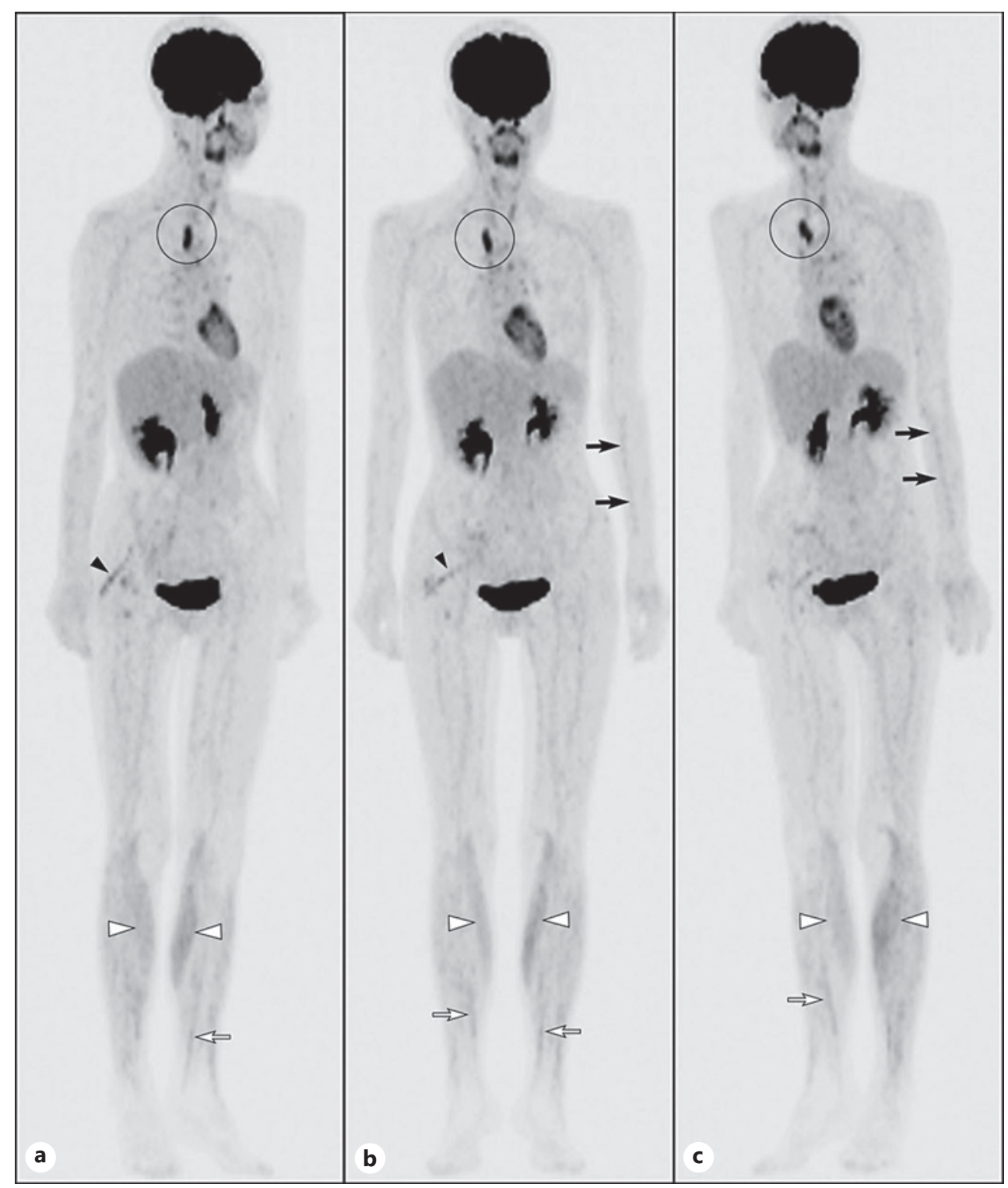

Fig. 1. ${ }^{18}$ F-FDG-PET before treatment. a Right oblique view. b Frontal view. $\mathbf{c}$ Left oblique view. Abnormal ${ }^{18}$ F-FDG accumulation is visualized in the mediastinal lymph node ( $\mathbf{a}-\mathbf{c}$; circles), left ulnar nerve (b, c; black arrows), right superior gluteal nerve (a, b; black arrowheads), bilateral tibial nerves (a-c; white arrows), and gastrocnemius muscles (a-c; white arrowheads). ${ }^{18}$ F-FDG-PET, ${ }^{18}$ F-fluorodeoxyglucose positron emission tomography.

the left ulnar nerve showed decreased motor nerve conduction velocity with conduction block; the bilateral tibial nerves showed a marked decrease in compound muscle action potential, which made accurate measurement of motor nerve conduction velocity difficult, but prolonged duration in the left tibial nerve suggested demyelination. The decreased compound muscle action potential in the bilateral peroneal nerves and the decreased sensory nerve action potential in the bilateral sural nerves indicated axonal involvement. Needle electromyography showed polyphasic and high-amplitude long-duration motor unit potentials in the right gluteus maximus, rectus femoris, and gastrocnemius. Magnetic resonance imaging showed no abnormal signal intensity in the upper and lower limbs. Histopathological examination of bone marrow aspiration and biopsy samples showed 
Table 1. Results of nerve conduction studies

\begin{tabular}{|c|c|c|c|c|c|c|c|c|c|}
\hline & \multirow[t]{2}{*}{$\mathrm{MCV}, \mathrm{m} / \mathrm{s}$} & \multicolumn{2}{|c|}{ CMAP, mV } & \multirow[t]{2}{*}{ CB } & \multirow{2}{*}{$\begin{array}{l}\mathrm{F} \text { wave } \\
\text { frequency, \% }\end{array}$} & \multirow{2}{*}{$\begin{array}{l}\text { FWCV, } \\
\mathrm{m} / \mathrm{s}\end{array}$} & \multirow{2}{*}{$\begin{array}{l}\mathrm{SCV}, \\
\mathrm{m} / \mathrm{s}\end{array}$} & \multicolumn{2}{|c|}{ SNAP, $\mu V$} \\
\hline & & distal & proximal & & & & & distal & proximal \\
\hline Median, L & 46.6 & 10.6 & 9.3 & - & 44 & 49.4 & 58.3 & 19.5 & $3.5^{\dagger}$ \\
\hline Median, R & 51.3 & 12.7 & 9.2 & - & 25 & 54.5 & 58.3 & 20.2 & 8.1 \\
\hline Ulnar, L & $34.5^{*}$ & 10.7 & 4.8 & + & 19 & 45.5 & 50.0 & 19.6 & $2.0^{\dagger}$ \\
\hline Ulnar, R & 55.6 & 13.9 & 14.1 & - & 100 & 57.4 & 53.0 & 26.3 & 15.6 \\
\hline Tibial, L & $15.6^{*}$ & $0.3^{*}$ & 0.08 & - & $\mathrm{N} / \mathrm{E}$ & N/E & $\mathrm{N} / \mathrm{A}$ & N/A & $\mathrm{N} / \mathrm{A}$ \\
\hline Tibial, R & $12.8^{*}$ & $0.6^{*}$ & 0.3 & - & $\mathrm{N} / \mathrm{E}$ & N/E & $\mathrm{N} / \mathrm{A}$ & N/A & $\mathrm{N} / \mathrm{A}$ \\
\hline Peroneal, L & 36.1 & $2.0^{*}$ & 1.6 & - & $\mathrm{N} / \mathrm{A}$ & $\mathrm{N} / \mathrm{A}$ & $\mathrm{N} / \mathrm{A}$ & $\mathrm{N} / \mathrm{A}$ & $\mathrm{N} / \mathrm{A}$ \\
\hline Peroneal, R & 35.0 & $0.5^{*}$ & 0.3 & - & $\mathrm{N} / \mathrm{A}$ & $\mathrm{N} / \mathrm{A}$ & N/A & $\mathrm{N} / \mathrm{A}$ & N/A \\
\hline Sural, L & $\mathrm{N} / \mathrm{A}$ & N/A & $\mathrm{N} / \mathrm{A}$ & $\mathrm{N} / \mathrm{A}$ & $\mathrm{N} / \mathrm{A}$ & $\mathrm{N} / \mathrm{A}$ & 35.9 & $\mathrm{~N} / \mathrm{A}$ & $2.1^{*}$ \\
\hline Sural, R & N/A & N/A & N/A & $\mathrm{N} / \mathrm{A}$ & N/A & N/A & 33.5 & N/A & $2.7^{*}$ \\
\hline
\end{tabular}

$\mathrm{MCV}$, motor conduction velocity; CMAP, compound muscle action potential; CB, conduction block; FWCV, F wave conduction velocity; SCV, sensory conduction velocity; SNAP, sensory nerve action potential; L, left; $\mathrm{R}$, right; N/E, not evoked; N/A, not available.

*Values represent below reference threshold.

tValues represent comparatively low values compared to the opposite site.

Fig. 2. Hematoxylin and eosin stain of mediastinal lymph node biopsy specimen. Noncaseating granulomas and a multinucleated giant cell are present (scale bar, $100 \mu \mathrm{m}$ ).

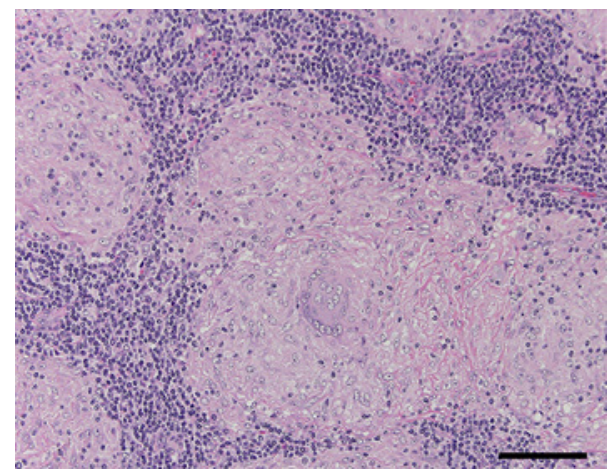

normocellular marrow without atypical cells. A left sural nerve biopsy indicated mild loss of myelinated fibers without cellular infiltration or granulomas. Surgical biopsy of the mediastinal lymph node which showed abnormal ${ }^{18} \mathrm{~F}$-FDG uptake revealed the presence of noncaseating epithelioid cell granulomas and the absence of lymphoma cells (Fig. 2). Ophthalmologic examination revealed peripheral anterior synechiae and cellular infiltration in the bilateral anterior chambers, consistent with uveitis. Gadolinium-enhanced myocardial cine magnetic resonance imaging showed delayed enhancement in the left ventricle, indicating inflammation and fibrosis in the interstitium of the myocardium, although there were no electrocardiographic or cardiac ultrasonographic abnormalities.

Based on the presence of noncaseating epithelioid granulomas in the mediastinal lymph node, uveitis, and cardiac inflammation, we diagnosed systemic sarcoidosis, rather than a sarcoid reaction or ML. We also diagnosed mononeuropathy multiplex as neurosarcoidosis, rather than neurolymphomatosis, because of slow progression, poor pain, and no atypical cells in the CSF, bone marrow, and lymph node. Radiological findings in the gastrocnemius muscles indicated sarcoid myopathy, although muscle biopsy was not performed. Treatment with oral prednisolone 


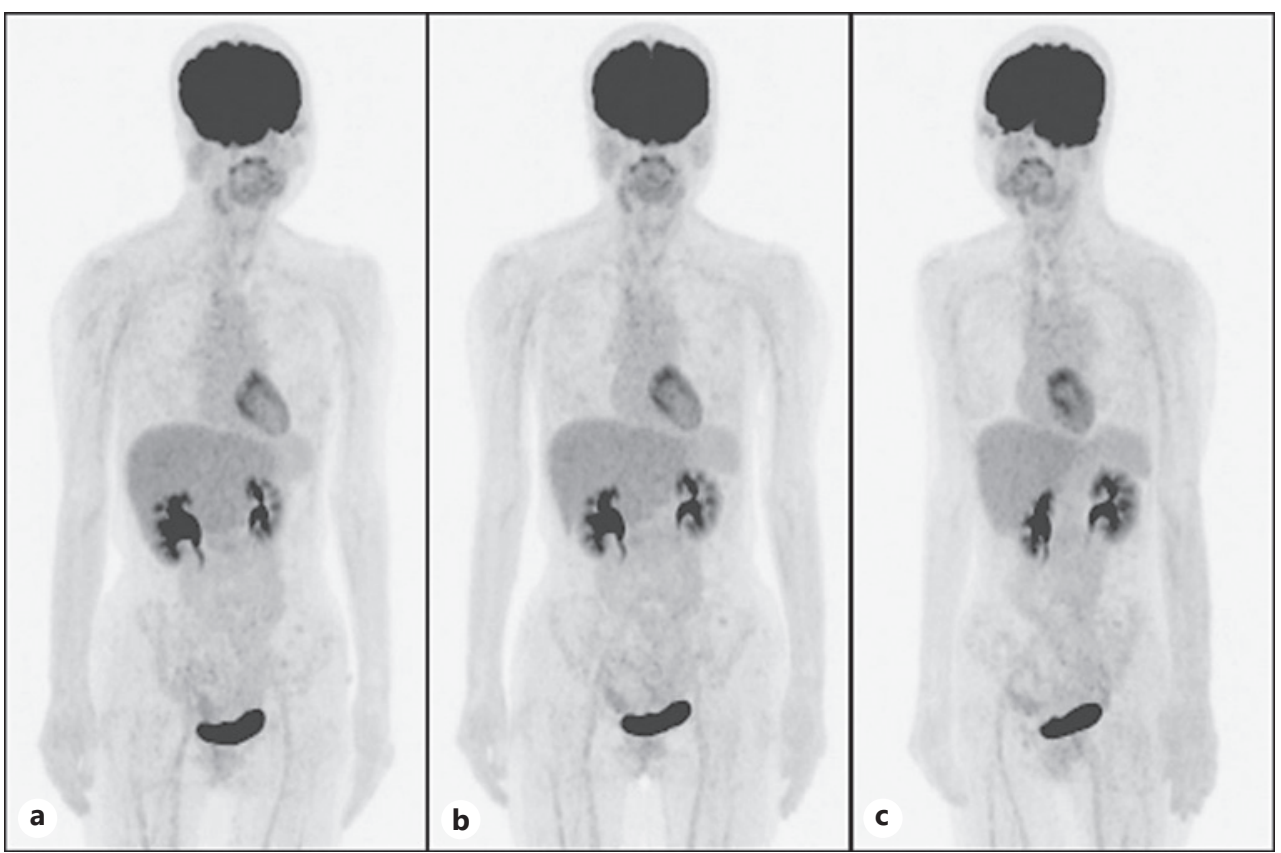

Fig. $3 .{ }^{18}$ F-FDG-PET after treatment. a Right oblique view. b Frontal view. c Left oblique view. ${ }^{18}$ F-FDG accumulation in the left ulnar and right superior gluteal nerves is no longer present 1.5 years after starting immunosuppressive therapy (the lower limbs were excluded from imaging). ${ }^{18} \mathrm{~F}$-FDG-PET, ${ }^{18} \mathrm{~F}$-fluorodeoxyglucose positron emission tomography.

$40 \mathrm{mg} /$ day was initiated, and her neurological manifestations gradually resolved. Subsequently, prednisolone was tapered to $15 \mathrm{mg} /$ day, and azathioprine (100 mg/day) was added. The serum sIL-2R level decreased to within the reference range $(346 \mathrm{U} / \mathrm{mL}) .{ }^{18} \mathrm{~F}-\mathrm{FDG}$-PET no longer showed accumulation of ${ }^{18} \mathrm{~F}$-FDG in the left forearm and right gluteal region (Fig. 3a-c); because the lower limbs were excluded from imaging, we could not evaluate the tibial nerves and gastrocnemius muscles. Three years after diagnosis, her neurological symptoms did not recur.

\section{Discussion}

We presented a case of neurosarcoidosis mimicking ML recurrence. Neurosarcoidosis was diagnosed based on mononeuropathy multiplex, uveitis, cardiac inflammation, and noncaseating epithelioid granulomas in the mediastinal lymph node. Treatment with high-dose steroids and immunosuppressants improved her neurological symptoms. Since sarcoid reaction and sarcoidosis are pathologically similar, sarcoid reaction for ML could not be completely ruled out in our case. However, the uveitis and cardiac inflammation were compatible to sarcoidosis, rather than sarcoid reaction of ML [8]. Furthermore, we concluded that mononeuropathy multiplex was caused by sarcoidosis and not by the recurrence of ML due to the absence of lymphoma cells in the pathological investigations, gradual clinical course, and long-term favorable outcome for corticosteroids and azathioprine. A definitive diagnosis of neurosarcoidosis could be made by peripheral nerve biopsy where ${ }^{18} \mathrm{~F}$-FDG was accumulated, but it was not possible because the sequelae of nerve biopsy were predicted. In a previous review of neurosarcoidosis, $71 \%$ of cases were diagnosed based on signs of inflammation in the nervous system in conjunction with positive histologic findings in a systemic lesion; only $2 \%$ of cases had positive histologic findings of 
sarcoidosis in a nervous system biopsy specimen [2]. Noncaseating granulomas were not observed in the biopsy specimen of the sural nerve, but they might be seen at a more proximal location from the biopsy site. The disappearance of accumulated ${ }^{18} \mathrm{~F}-\mathrm{FDG}$ in the ulnar and superior gluteal nerves after immunotherapy supported the diagnosis of sarcoidosis lesions in these peripheral nerves.

As described earlier, sarcoidosis following ML is less common than sarcoidosis-lymphoma syndrome [7]. In terms of the pathogenesis of sarcoidosis following ML, an excessive immune response against lymphoma cells has been implicated in the formation of sarcoid reaction [6], which may facilitate the development of sarcoidosis. Furthermore, rituximab, which was administered to our case when ML relapsed, has been reported to elicit repopulation of peripheral B cells and re-establishment of a naive B-cell pool, leading to the development of sarcoidosis [9]. We hypothesize that the immune response against lymphoma cells and immune re-establishment by rituximab promoted the development of sarcoidosis in this case, but these immune reactions usually occur within 1 year [8]. In the previous report, most cases of sarcoidosis following ML occur within 2 years, but in rare cases, sarcoidosis develops $>10$ years after ML [8]. The age at onset of sarcoidosis following ML tends to be older than idiopathic sarcoidosis, with the median age of the former being 49 (range, 23-71) years [8] and the latter being 31 years [10]. In our case, the patient was 65 years old when she presented with sarcoidosis, which is atypical for idiopathic sarcoidosis. We could not elucidate the mechanisms involved in the development of sarcoidosis after long-term remission of ML, so further investigations need to be performed.

Several biomarkers support the diagnosis of sarcoidosis. The serum and CSF sIL-2R levels are not necessarily useful for differentiating sarcoidosis from lymphoma [11, 12]. Although the serum ACE level is elevated in sarcoidosis patients, it is also elevated in patients with Hodgkin's lymphoma, hence its low specificity [13]. The CSF ACE level is of little clinical value for diagnosing neurosarcoidosis [4]. These biomarkers only play a supplementary role in the diagnosis of sarcoidosis.

${ }^{18}$ F-FDG-PET has been widely used for tumor diagnosis, differential diagnosis, staging, follow-up, therapy planning, and prognosis. However, ${ }^{18}$ F-FDG-PET cannot differentiate between sarcoidosis and ML because no statistical difference was seen in SUVmax [14]. On the other hand, ${ }^{18}$ F-FDG-PET is useful for diagnosing asymptomatic sarcoidosis during the follow-up for lymphoma [8]. In addition, ${ }^{18} \mathrm{~F}$-FDG-PET is valuable for identifying lesions amenable to biopsy in patients with multisystem sarcoidosis and evaluating response to treatment [3]. In our patient, we elected to perform a biopsy of the mediastinal lymph node with abnormal ${ }^{18} \mathrm{~F}$-FDG accumulation. Our experience indicates that ${ }^{18} \mathrm{~F}$-FDG-PET may be helpful to detect undiagnosed mononeuropathy multiplex. Follow-up ${ }^{18} \mathrm{~F}$-FDG-PET showed the disappearance of uptake of ${ }^{18} \mathrm{~F}-\mathrm{FDG}$ in the ulnar and superior gluteal nerves, confirming that it was also useful for evaluating treatment response.

In conclusion, due to its similarity to neurolymphomatosis, the diagnosis of neurosarcoidosis should be made from tissue biopsy in addition to clinical presentations and radiological findings. Recurrence of ML is not definitively ruled out, so regular follow-ups should be performed.

\section{Acknowledgements}

We thank Dr. Koh-suke Nishi and Dr. Hiroki Iwanishi at the Department of Ophthalmology, Wakayama Medical University, for ophthalmologic examination and Dr. Yoshifumi Iwahashi and Dr. Shin-ichi Murata at the Department of Pathology, Wakayama Medical University, for pathological examination.

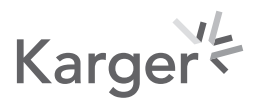




\section{Statement of Ethics}

The research was conducted ethically in accordance with the World Medical Association Declaration of Helsinki. The patient provided written informed consent for publication of this article including images. The study is exempt from ethical committee approval because this is a case report study and does not include the experiment on animal and human subjects.

\section{Conflict of Interest Statement}

The authors have no conflicts of interest to declare.

\section{Funding Sources}

The authors have not declared a specific grant for this research from any funding agency in the public, commercial, or not-for-profit sectors.

\section{Author Contributions}

K.M., J.K., and J.T. were involved in the diagnosis and care of the patient. K.M. drafted the manuscript and reviewed literature. J.K. and J.T. revised and updated the manuscript. H.I. provided the idea of the manuscript and supervised the study. All of the authors read and approved the final version.

\section{Data Availability Statement}

All data generated or analyzed during this study are included in this article. Further inquiries can be directed to the corresponding author.

\section{References}

1 Culver DA, Judson MA. New advances in the management of pulmonary sarcoidosis. BMJ. 2019 Oct;367:15553.

2 Leonhard SE, Fritz D, Eftimov F, van der Kooi AJ, van de Beek D, Brouwer MC. Neurosarcoidosis in a tertiary referral center: a cross-sectional cohort study. Medicine. 2016 Apr;95(14):e3277.

3 Wang Y, Andrews J, Jenkins Colon P, Wundes A. FDG-PET abnormalities leading to the diagnosis of an unusual case of probable neurosarcoidosis. Neurol Neuroimmunol Neuroinflamm. 2018 Nov;5(6):e506.

4 Bridel C, Courvoisier DS, Vuilleumier N, Lalive PH. Cerebrospinal fluid angiotensin-converting enzyme for diagnosis of neurosarcoidosis. J Neuroimmunol. 2015 Aug;285:1-3.

5 Brincker H, Wilbek E. The incidence of malignant tumours in patients with respiratory sarcoidosis. Br J Cancer. 1974 Mar;29(3):247-51.

6 Brincker H. The sarcoidosis-lymphoma syndrome. Br J Cancer. 1986 Sep;54(3):467-73.

7 Cohen PR, Kurzrock R. Sarcoidosis and malignancy. Clin Dermatol. 2007 May-Jun;25(3):326-33.

8 London J, Grados A, Fermé C, Charmillon A, Maurier F, Deau B, et al. Sarcoidosis occurring after lymphoma: report of 14 patients and review of the literature. Medicine (Baltimore). 2014 Nov; 93(21):e121.

9 Ueda-Hayakawa I, Tanimura H, Osawa M, Iwasaka H, Ohe S, Yamazaki F, et al. Elevated serum BAFF levels in patients with sarcoidosis: association with disease activity. Rheumatology. 2013 Sep;52(9):1658-66.

10 Horwitz O, Payne PG, Wilbek E. Epidemiology of sarcoidosis in Denmark. Dan Med Bull. 1967 Jun;14(7):17882.

11 Kita T, Watanabe S, Yano F, Hayashi K, Yamamoto M, Iwasaki Y, et al. Clinical significance of the serum IL-2R level and Ga-67 scan findings in making a differential diagnosis between sarcoidosis and non-Hodgkin's lymphoma. Ann Nucl Med. 2007 Nov;21(9):499-503. 
12 Petereit HF, Reske D, Tumani H, Jarius S, Markus Leweke F, Woitalla D, et al. Soluble CSF interleukin 2 receptor as indicator of neurosarcoidosis. J Neurol. 2010 Nov;257(11):1855-63.

13 Koca E, Haznedaroglu IC, Uner A, Sayinalp N, Saglam AE, Goker H, et al. Angiotensin-converting enzyme expression of the lymphoma-associated macrophages in the lymph nodes of Hodgkin's disease. J Natl Med Assoc. 2007 Nov;99(11):1243-7, 1246-7.

14 Yu C, Xia X, Qin C, Sun X, Zhang Y, Lan X. Is SUVmax helpful in the differential diagnosis of enlarged mediastinal lymph nodes? A pilot study. Contrast Media Mol Imaging. 2018. eCollection. 